\title{
Correction of Minor-Form and Microform Cleft Lip Using Modified Muscle Overlapping with a Minimal Skin Incision
}

\author{
Min Chul Kim ${ }^{1}$, Dong Hun $\mathrm{Choi}^{1}$, Sung Gun Bae ${ }^{2}$, Byung Chae Cho ${ }^{1}$ \\ ${ }^{1}$ Department of Plastic and Reconstructive Surgery, Kyungpook National University School of Medicine, Daegu; ${ }^{2}$ BL Plastic Surgery Clinic, \\ Daegu, Korea
}

Background In treating minor-form or microform cleft lip, obtaining an optimal result is a challenge because of the visible scarring caused by traditional surgery. We present a refined method using muscle overlapping with a minimal skin incision in patients younger than 3 years, a group characterized by thin muscle.

Methods The surgical technique involves restoration of the notched vermillion using Z-plasty, formation of the philtral column using overlapping of an orbicularis oris muscle flap through an intraoral incision, and correction of the cleft lip nasal deformity using a reverse- $\mathrm{U}$ incision and $\mathrm{V}-\mathrm{Y}$ plasty. A single radiologist evaluated ultrasonographic images of the upper lip.

Results Sixty patients were treated between September 2008 and June 2014. The age at the time of operation ranged from 6 to 36 months (mean, 26 months). The follow-up period ranged from 8 to 38 months (mean, 20 months) in minor-form cases and from 14 to 64 months (mean, 37 months) in microform cases. A notched cupid's bow was corrected in 10 minor-form cases and 50 microform cases. Ultrasonographic images were obtained from 3 patients with minor-form cleft lip and 9 patients with microform cleft lip 12 months after surgery. The average muscle thickness was $4.5 \mathrm{~mm}$ on the affected side and $4.1 \mathrm{~mm}$ on the unaffected side.

Conclusions The advantages of the proposed procedure include the creation of an anatomically natural philtrum with minimal scarring. This method also preserves the continuity and function of the muscle and provides sufficient augmentation of the philtral column and nostril sill.

\section{Keywords Cleft lip / Surgical flaps / Cicatrix}

Correspondence: Byung Chae Cho Department of Plastic and Reconstructive Surgery, Kyungpook National University School of Medicine, 130 Dongdeok-ro, Jung-gu, Daegu 41944, Korea

Tel: +82-53-420-5691

Fax: +82-53-425-3879

E-mail: bccho@knu.ac.kr

\section{INTRODUCTION}

Minor-form and microform cleft lip (CL) are differentiated primarily by the extent of the cleft. The difference between the normal subnasale-to-cupid's bow peak distance and the cleftside subnasale-to-cupid's bow peak distance is equal to the infe- rior displacement of the peak necessary to construct a level cupid's bow. If the difference is $3 \mathrm{~mm}$ or more, the cleft is defined as minor-form, and if less than $3 \mathrm{~mm}$, as microform [1]. In most cases of minor-form and microform CL, normal anatomical structures remain present, with very slight displacement.

Various techniques have been described to repair minor-form 
and microform CL depending on the severity of the deformity [1-10]. The objectives of the surgical repair of minor-form and microform CL are to eliminate any notch of the vermillion, to correct the drooping or flattened ala, and to restore muscle continuity [2]. The reestablishment of orbicularis oris muscle (OOM) continuity is important aesthetically for lip symmetry during repose, is important functionally during movement, and is essential to creating a natural philtral column [11]. To achieve muscle continuity with a minimal scar in the treatment of minor-form and microform $\mathrm{CL}$, a method using vertical interdigitation of the OOM to create a philtrum through an intraoral incision, thereby avoiding an external scar, and Z-plasty to correct the notched vermillion-cutaneous junction was previously introduced and refined $[2,3]$. In patients younger than 3 years, whose OOM is thin and difficult to split, an overlapping method was also described [3].

However, some patients present lateral muscle bulging in the puckering state despite presenting a natural philtral column in the resting state. During follow-up, to ameliorate these problems, we modified the conventional surgical method and applied this method to such patients, leaving minimal scarring. Herein, we report this new technique for the treatment of minor-form and microform CL using muscle overlapping through an intraoral incision with a minimal skin incision. In addition, we compared postoperative OOM thickness between the reconstructed and unaffected sides using ultrasonography.

\section{METHODS}

A total of 60 patients, 10 with minor-form CL and 50 with microform CL, were treated between September 2008 and June 2014. These patients were diagnosed according to the classifica- tion of Yuzuriha and Mulliken [1]. The resting state was defined as the state in which patients did not have a facial expression or speak, and maximal puckering was defined as the state in which patients had maximal tension in the OOM, and therefore a maximally puckered mouth. In both the resting and maximal puckering states, the thickness of the OOM was measured bilaterally with ultrasonography, using a linear array transducer in parallel with the occlusal plane.

\section{Correction of the notch of cupid's bow in minor-form or microform CL}

Minor-form CL, with a notched vermillion-cutaneous junction extending $3 \mathrm{~mm}$ or more above the normal cupid's bow peak, was repaired with double unilimb Z-plasty instead of traditional rotation-advancement repair on the external lip [4]. The procedure included unilimb Z-plasty both at the vermillion-cutaneous junction and at the vermillion-mucosal junction. If more inferior displacement was required on the cleft side of the cupid's bow peak, an additional triangular flap was inserted on the cutaneous upper lip (Fig. 1). Microform CL, with the medial vermillion-cutaneous point less than $3 \mathrm{~mm}$ above the normal cupid's bow peak, was repaired with Z-plasty, as described by Onizuka et al. [5] and Millard [12]. The incision was placed along the vermillion. The vermillion and the skin inserted in the vermillion were transposed by a Z-plasty (Fig. 2). U-shaped defects of the vermillion with loss of the mucocutaneous ridge were treated with simple excision or a rotation vermillion mucosal flap [2].

\section{Correction of depressed groove on the philtral column}

We developed a modified version of a previously reported technique [2]. A full-thickness intraoral incision was made down to

Fig. 1. Double unilimb Z-plasty of minor-form cleft lip

(A) Preoperatively, (B) intraoperatively, and (C) postoperatively. The medial and lateral orbicularis oris muscle flaps were elevated and overlapped through an intraoral incision, as shown in Fig. 3.

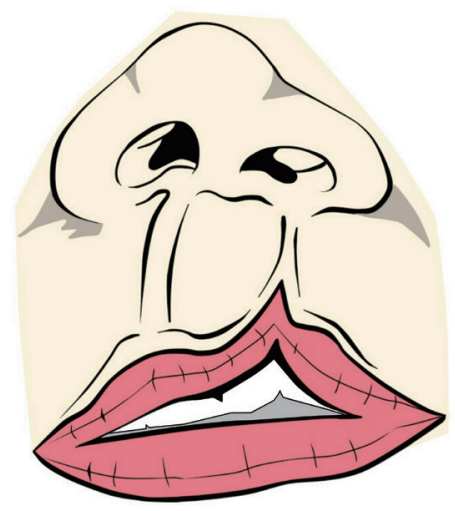

(A)
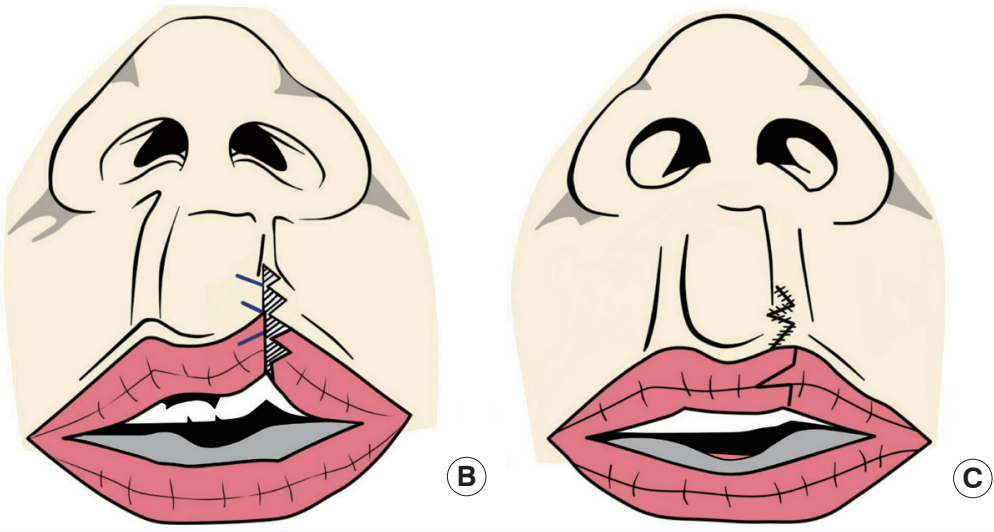


\section{Fig. 2. Z-plasty of microform cleft lip}

(A) Preoperatively, (B) intraoperatively, and (C) postoperatively.

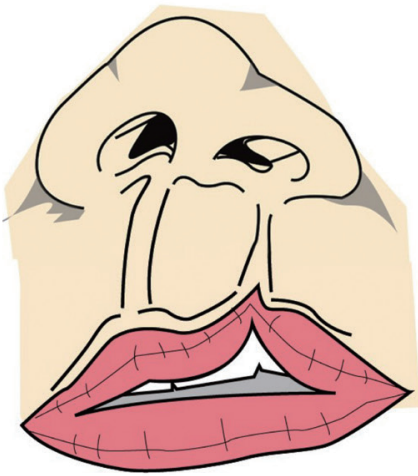

(A)

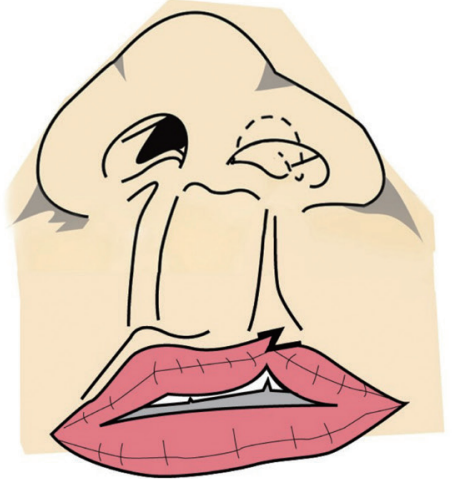

(B)

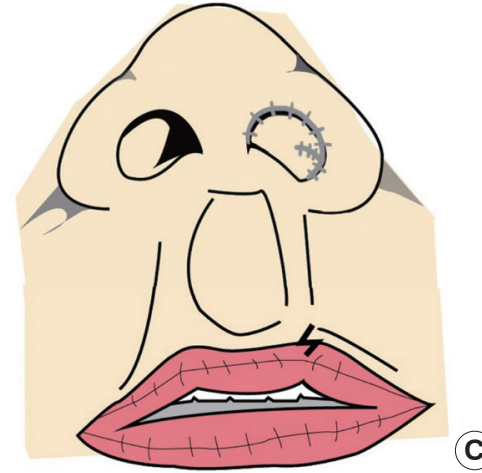

the mucosa and the posterior portion of the muscle. The remaining muscle was dissected with blunt scissors. The muscle was carefully dissected down to the dermis of the depressed groove. Once the dermis was identified, the muscle was detached and freed $5 \mathrm{~mm}$ medially and 3-5 mm laterally from the dermis. Occasionally, in some of the minor-form cases, lateral lip bulging was observed preoperatively on the cleft side. In such cases, wider dissection might be required. To prevent lateral muscle bulging in the puckering state despite the presence of a natural-appearing philtral column in the resting state, a lateral muscle flap was firmly sutured to the dermis $1 \mathrm{~mm}$ lateral to the philtral dimple, a medial muscle flap was sutured to a point beneath the expected philtral column, and the lateral muscle flap was dissected less than in the original technique (i.e., less than 5 $\mathrm{mm}$ ), in order to prevent lateral bulging in the maximal puckering state. Additionally, each muscle flap in the vermillion was sutured in an end-to-end fashion to prevent vermillion bulging. This modified overlapping method is helpful in young patients, particularly those younger than 36 months, whose muscle is thin and difficult to split and interdigitate. Typically, 3 or 4 sutures were needed for the overlapping method. It is important to make a tight, large-bite suture in the muscle in the alar base to correct the depressed nostril sill. Any excess oral mucosa after overlapping of the muscle was trimmed and closed with 5-0 Vicryl (Fig. 3).

\section{Correction of the CL nasal deformity}

The CL nasal deformity was corrected using a reverse-U incision and V-Y plasty [2,13-15].

The cleft lower lateral cartilage was fixed to the normal lower lateral cartilage and the reverse-U flap was advanced medially and superiorly. Two or three alar transfixion sutures were placed in the vestibular skin area.
Fig. 3. Schematic representation of the overlapping technique

(A) Preoperatively, (B) intraoperatively, and (C) postoperatively. Note the red dots indicating where the lateral muscle flap was sutured to the dermis, $1 \mathrm{~mm}$ lateral to the philtral dimple. The medial muscle flap was fixed to the lateral muscle flap, just below the expected line of the newly created philtral column.

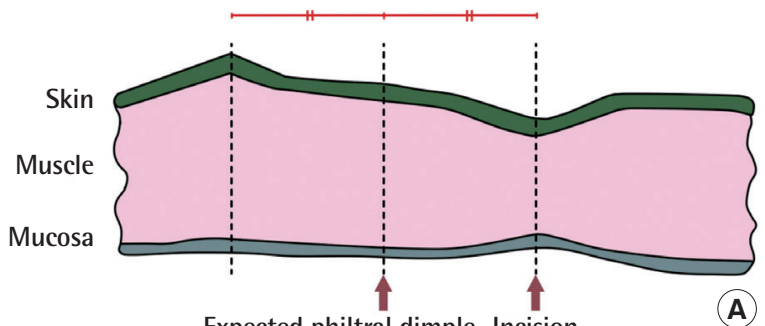

Expected philtral dimple Incision

(A)
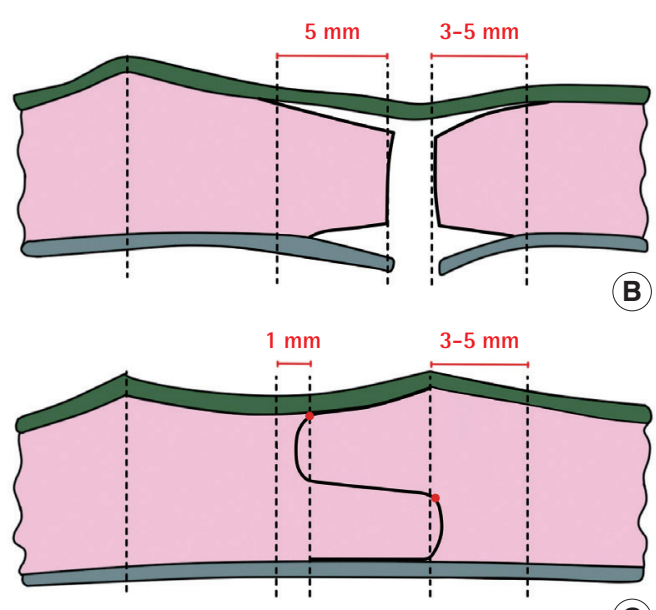

(C)

\section{Assessments}

A visual analog scale was used to assess the outcome of the corrected depressed groove on the philtral column. The prominence of the new philtral column was evaluated using a 3-point grading scale, with 1 indicating a conspicuous new column, as 


\section{Fig. 4. Six-month-old patient with minor-form cleft lip}

(A) Preoperative views show a notched vermillion-cutaneous junction extending $3 \mathrm{~mm}$ above the normal cupid's bow peak with vermillion deficiency and a depressed groove on the philtral column. (B) Design of double lateral triangles on both cutaneous and vermillion skin. (C) For muscle overlapping, the medial and lateral muscle flaps were detached from the oral mucosa and completely exposed. The forceps are shown grasping the detached lateral orbicularis oris muscle flap. (D) Immediate postoperative frontal view shows the newly created philtral column on the cleft side and correction of the notched cupid's bow with a minimal incision.
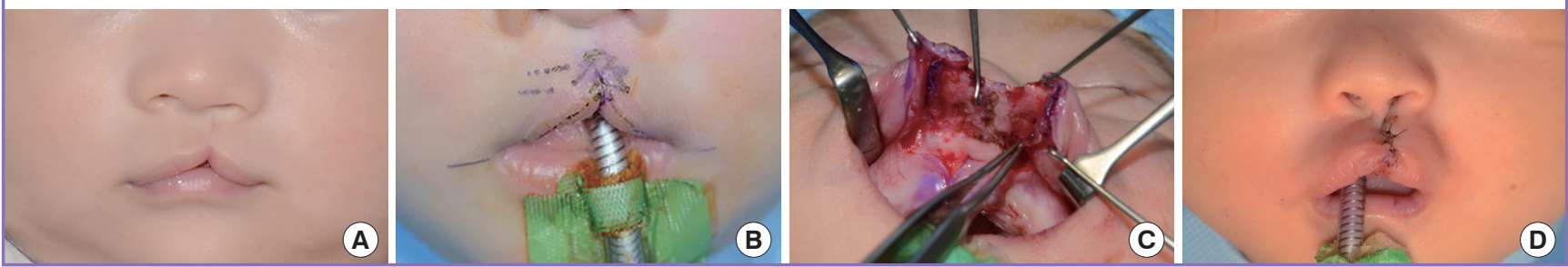

Fig. 5. Six-month-old child with minor-form cleft lip

A notched cupid's bow (3 mm or more above the normal cupid's bow peak), depressed groove on the philtral column, and vermillion deficiency on the left side. (A) Preoperative view. In this patient, double unilimb Z-plasty and overlapping of the muscle were performed. (B) Thirty-eightmonth postoperative view. (C) Postoperative view when puckering the lips.
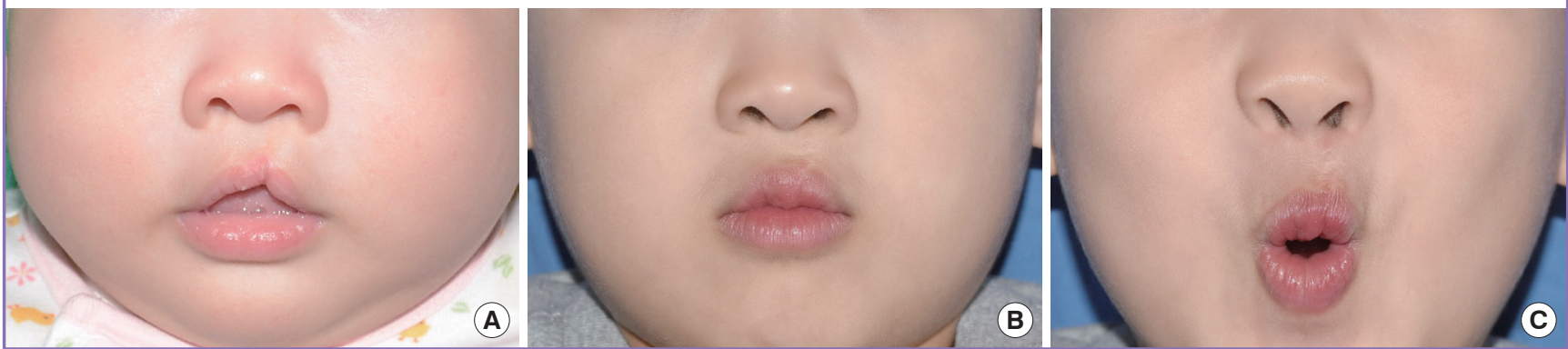

Fig. 6. Six-month-old child with microform cleft lip

A notched cupid's bow (less than $3 \mathrm{~mm}$ above the normal cupid's bow peak), depressed groove on the philtral column, vermillion deficiency, and cleft lip nasal deformity on the left side. (A) Preoperative view. In this patient, Z-plasty and overlapping of the muscle on the lip, reverse- $U$ incision, $V-Y$ plasty, and alar base advancement on the nose were performed. (B) Thirty-month postoperative view.

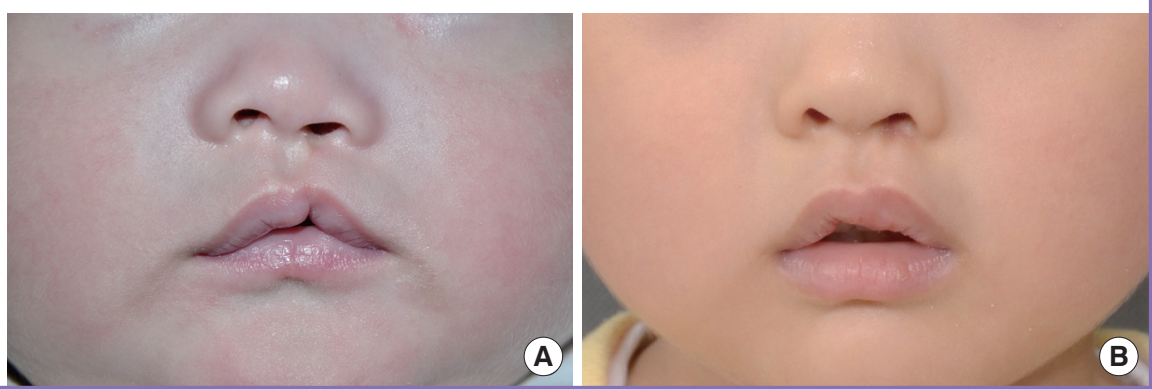

prominent as the normal side, 2 indicating no definite new philtral column, and 3 indicating no improvement. Ultrasonographic measurements were performed by a single radiologist using a linear array transducer. The OOM thickness in the resting state and maximal puckering states, in parallel with the occlusal plane and focusing on the center of the lip, was compared between the unaffected and affected sides at least 12 months postoperatively.

\section{RESULTS}

Ten patients with minor-form CL (Fig. 4) and 50 with micro- form CL underwent this procedure with no clinical complications (Figs. 5, 6). The age at the time of operation ranged from 6 to 36 months, with an average of 26 months. The male-to-female ratio was 3:1, and the left-to-right ratio was 3:1. The follow-up period ranged from 8 to 38 months in minor-form cases, with an average of 20 months, and from 14 to 64 months in microform cases, with an average of 37 months. An average of 50 minutes was required to perform the operation, and 5 days for the wound from the operation to heal. The results were satisfactory in all patients. Fifty-eight patients were assigned a score of 1 . However, 5 of these 58 patients presented lateral bulging in the 


\section{Fig. 7. Ultrasound image of the philtrum}

Muscle appears as a hypoechogenic (dark) line between the echogenic (light) areas of connective tissue. (A) Philtrum of the non-cleft side. (B) Philtrum of the cleft side.
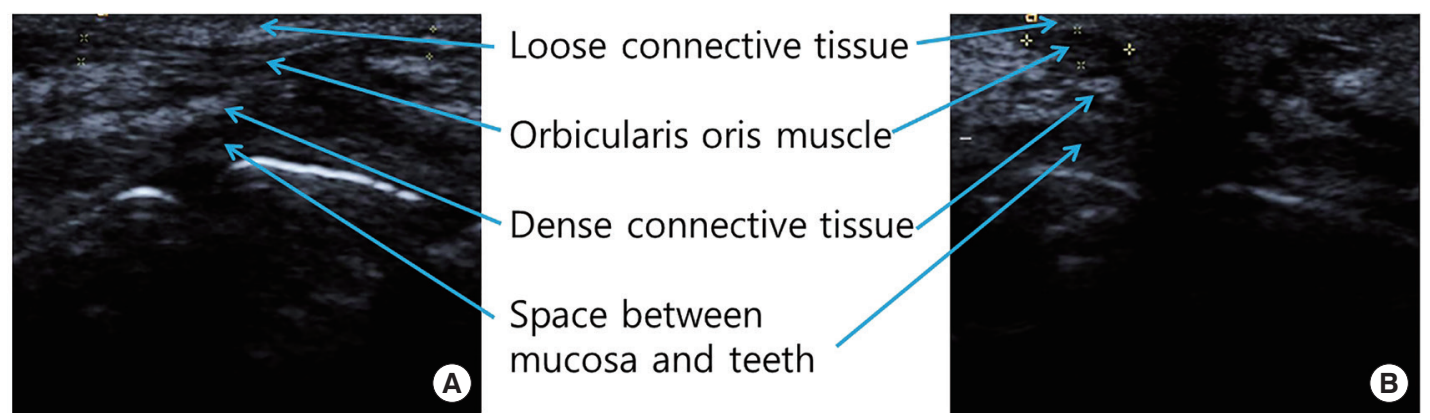

puckering state despite the presence of a natural reconstructed philtral column in the resting state. A possible cause for this outcome was over dissection of the lateral muscle flap and overcorrection of the overlapped muscle contents in the lateral part of the newly created philtral column. Two patients were assigned a score of 2. A possible cause for the absence of a definitive new philtral column was deficiency of the overlapped muscle content and partial disruption of the overlapped muscle flap, attributable to the early active movement of the muscle before wound healing. The notched cupid's bow was corrected by double unilimb Z-plasty in the 10 minor-form CL patients and by Zplasty in the 50 microform CL patients. In all cases, no operation scar was visible on the depressed philtral groove on the cleft side, and the notched cupid's bow was corrected.

\section{Complementary studies}

Ultrasonographic images of the CL surgical site were obtained in 3 patients with minor-form CL and 9 patients with microform CL 12 months after surgery. On ultrasound, the muscle appeared as a hypoechogenic area within surrounding connective tissues. The average muscle thickness was $4.5 \mathrm{~mm}$ on the affected side and $4.1 \mathrm{~mm}$ on the unaffected side in the resting state, and $4.8 \mathrm{~mm}$ on the affected side and $4.5 \mathrm{~mm}$ on the normal side in maximal puckering. These results indicate that the thickness of the reconstructed muscle was similar to or slightly greater than the thickness on the normal side. Ultrasound images indicated that the newly proposed surgical method led to an improved shape of the philtral ridge not only in the resting state, but also at maximal puckering (Fig. 7).

\section{DISCUSSION}

Microform CL appears to involve a 'minor' vermillion notch, but the underlying defect is more extensive. The thin skin in this area camouflages a discontinuity in the ring of the OOM. Even in cases of minor incomplete cleft, a groove on the skin was found to protrude from the cleft peak upward to the threshold of the nostril in autopsy studies, corresponding to a deficient muscular ring within the lip [16]. The apparent minor vermillion notch within the mucocutaneous border and the firm ridge stretching to the nostril floor may present surface findings secondary to the discontinuity of the OOM [17]. Therefore, muscle reconstruction is vital for optimal repair. The primary aim of cleft deformity correction is to reproduce the aesthetic appearance and function of the upper lip, including OOM and philtral ridge reconstruction, notched cupid's bow correction, and nasal deformity repair, with a minimal cutaneous scar [18].

Recently, Yuzuriha and Milliken [1] classified smaller variations of unilateral incomplete cleft deformities into 3 categories (minor-form, microform, and mini-microform), and described different operative techniques for each type. In minor-form and microform clefts, the philtral ridge is formed by repair of the muscular diastasis. The OOM is divided along the line of the cutaneous furrow and reapposed with everting mattress sutures, but this elevation along the philtral column is often insufficient, necessitating a dermal graft to augment the philtral ridge in the primary repair and/or secondary revisions. However, these grafting techniques are unpredictable because of the potential for resorption of the materials [6].

Yuzuriha and Milliken [1] defined a medial side notch of the vermillion exceeding $3 \mathrm{~mm}$ above the normal cupid's bow peak as a minor-form cleft, and $3 \mathrm{~mm}$ as the lowest technical limit for unilimb Z-plasty, adding that the standard rotation-advancement principle is preferable for the above case. However, the authors of the present study used double unilimb Z-plasty with a minimal skin incision and the OOM overlapping method for minor-form CL. This method reduced the scar size of the traditional rotation-advancement technique and allowed formation 
of the philtral column.

To reconstruct the OOM with little or no signs of a cutaneous scar, Desrosiers et al. [6] described a combined technique to repair microform $\mathrm{CL}$ with intraoral muscle interdigitation, modified from the Furlow, Mulliken, and Cutting techniques. In this technique, transverse interdigitation of the OOM via an intraoral approach is applied. With this technique, the OOM is incised and dissected, and then the muscle on each side is split transversely into 3 muscle slips. The muscle slips are interdigitated in a basket-weave pattern and secured to the dermis on either side of the philtral column. Notably, only philtral column volume is improved by this anchoring. However, in patients younger than 3 years who do not have a thick OOM, splitting of the orbicularis for the interdigitation technique is difficult and could cause fibrosis because of muscle damage. In such cases, we used vertical overlapping of the OOM flap by dissecting it from the skin and oral mucosa.

More recently, Oyama et al. [7] further emphasized the importance of identification and utilization of the cupid's bow peak on the lateral side in minor-form/microform CL repair, by defining the peak of the curve on the lateral lip as the cupid's bow peak on the cleft side and suggesting that this structure be preserved to achieve a more natural outcome. Thus, in this technique, a modified incision line to maintain the natural curve is made, and a small triangular flap located above the cupid's bow peak of the lateral lip is designed and inserted toward the deepest point of the philtrum. However, Oyama et al. did not describe the reconstruction of the philtral ridge.

The reestablishment of OOM continuity to create a natural philtral column with minimal scarring on the upper lip, the primary objective of surgical correction, has remained a challenge $[2,3,15,19,20]$. OOM repair was rarely mentioned in minorform CL surgery until the first report of a method for the correction of minor-form and microform CL through vertical interdigitation of the orbicularis in 2004 [2]. In the present study, the authors describe reconstruction of the OOM of patients with minor-form $\mathrm{CL}$ intraorally via an overlapping method and the use of double unilimb Z-plasty, rather than a conventional advancement-rotation flap, to minimize scarring after surgery.

This proposed procedure restores muscle continuity through an intraoral incision to avoid an external scar on the upper lip, and provides sufficient augmentation of the philtral column by vertical overlapping of the OOM [2]. Overlapping of the medial and lateral muscle flaps also maintains muscle continuity and function and avoids any interference with muscle motion. The thickness of the newly created philtral column is initially overcorrected and typically demonstrates a mild to moderate decrease during the first 6 months postoperatively, due to lip move- ment. There was no further change in the height of the new philtral column in any of the patients during long-term follow-up, and in most cases the final thickness was comparable to a normal philtral column. Meticulous sutures between the muscle flaps are recommended during vertical overlapping of the muscle to avoid wound disruption and to maintain maximal thickness of the philtral column. In particular, this technique is very effective for augmenting the depressed nostril sill and alar base by overlapping the muscle flaps without using filling materials.

Although the previously described technique can achieve good results, some modifications are still needed. To prevent the occurrence of lateral muscle bulging in the puckering state, despite a natural-appearing philtral column in the resting state, the lateral muscle flap should be fixed to the dermis just $1 \mathrm{~mm}$ lateral to the philtral dimple. Moreover, the dissection is restricted to $5 \mathrm{~mm}$ laterally, and the medial muscle flap is overlapped and sutured to just below the expected philtral column, rather than the base of the lateral muscle flap. Another potential problem is minor muscle bulging in the vermillion, which is caused by the volume of overlapped muscle. In such cases, the muscle flap in the vermillion should not be overlapped, but is instead closed in an end-to-end fashion to avoid this shortcoming. In this article, we introduced a modified technique for the correction of minor-form and microform CL using muscle overlapping through an intraoral incision, and obtained good results both functionally and aesthetically over a long-term follow-up.

Ultrasonography is advantageous for observing the anatomical structure of the upper lip in that it enables more rapid image acquisition than magnetic resonance imaging, and has high resolution [21]. A method to distinguish normal OOM from OOM with scarring on ultrasound images has been previously reported [21]. Moreover, muscle defects due to scar formation can be quantitatively measured using ultrasonography. Kim et al. [22] examined the correlation between OOM defects and philtral appearance using ultrasonography and found that the convexity of the philtral ridge decreased as muscle defects increased in the resting state. These findings suggest that CL muscle defects may lead to a defect or, in the worst case, a dent of the philtral ridge, since the bilateral OOM forms the philtral ridge, and that secure reconstruction of the OOM could result in the desired outcomes of CL surgery.

In conclusion, we modified the conventional technique and applied this new surgical method to patients with minor-form and microform CL. The major advantage of this procedure is the creation of an anatomically natural philtrum with minimal external scarring. Especially in minor-form $\mathrm{CL}$ patients, muscle rearrangement and philtrum formation can be achieved by using the OOM overlapping method through a minimal intraoral 
incision instead of the conventional rotation-advancement technique.

\section{REFERENCES}

1. Yuzuriha S, Mulliken JB. Minor-form, microform, and minimicroform cleft lip: anatomical features, operative techniques, and revisions. Plast Reconstr Surg 2008;122:148593.

2. Cho BC. New technique for correction of the microform cleft lip using vertical interdigitation of the orbicularis oris muscle through the intraoral incision. Plast Reconstr Surg 2004;114:1032-41.

3. Cho BC. Refined new technique for correction of the minor-form, microform cleft lip and minor-form bilateral cleft lip through the intraoral incision and long-term results. Plast Reconstr Surg 2011;127:781-3.

4. Mulliken JB. Double unilimb Z-plastic repair of microform cleft lip. Plast Reconstr Surg 2005;116:1623-32.

5. Onizuka T, Hosaka Y, Aoyama R, et al. Operations for microforms of cleft lip. Cleft Palate Craniofac J 1991;28:293300.

6. Desrosiers AE 3rd, Kawamoto HK, Katchikian HV, et al. Microform cleft lip repair with intraoral muscle interdigitation. Ann Plast Surg 2009;62:640-4.

7. Oyama A, Funayama E, Furukawa H, et al. Minor-form/microform cleft lip repair: the importance of identification and utilization of Cupid bow peak on the lateral lip. Ann Plast Surg 2014;72:47-9.

8. Cosman B, Crikelair GF. The minimal cleft lip. Plast Reconstr Surg 1966;37:334-40.

9. Sigler A, Ontiveros DS. Nasal deformity and microform cleft lip in parents of patients with cleft lip. Cleft Palate Craniofac J 1999;36:139-43.

10. Koh KS, Hwang CH, Kim EK. Modified design of Cupid's bow in the repair of unilateral microform cleft lip: in case of deficient distance between the midline and the cleft side Cupid's bow peak. J Craniofac Surg 2009;20:1367-9.

11. Schlezinger NS. A study of the oral (orbicularis oris) reflex. Psychiatric Quarterly 1938;12:629-49.

12. Millard DR. Cleft craft: the evolution of its Surgery. Vol I: the unilateral deformity. Boston: Little Brown; 1976.

13. Tajima $S$, Maruyama $M$. Reverse-U incision for secondary repair of cleft lip nose. Plast Reconstr Surg 1977;60:256-61.

14. Cronin TD. Lengthening columella by use of skin from nasal floor and alae. Plast Reconstr Surg Transplant Bull 1958; 21:417-26.

15. Cho BC, Baik BS. Correction of cleft lip nasal deformity in Orientals using a refined reverse-U incision and V-Y plasty. Br J Plast Surg 2001;54:588-96.

16. Fara M. Anatomy and arteriography of cleft lips in stillborn children. Plast Reconstr Surg 1968;42:29-36.

17. Thaller SR, Lee TJ. Microform cleft lip associated with a complete cleft palate. Cleft Palate Craniofac J 1995;32:24750.

18. Lee TJ. Clinical manifestation of microform cleft lip (Abstract). Presented at: the Korean Cleft Palate-Craniofacial Association Symposium; 2003 Apr 19; Kwangju, Korea.

19. Cho BC, Park JW, Baik BS. Correction of severe secondary cleft lip nasal deformity using a composite graft: current approach and review. Ann Plast Surg 2002;48:131-7.

20. Cho BC. Correction of unilateral cleft lip nasal deformity in preschool and school-aged children with refined reverse- $U$ incision and V-Y plasty: long-term follow-up results. Plast Reconstr Surg 2007;119:267-75.

21. van Hees NJ, Thijssen JM, Huyskens RW, et al. Quantitative ultrasound imaging of healthy and reconstructed cleft lip: a feasibility study. Cleft Palate Craniofac J 2007;44:261-8.

22. Kim SW, Jeong YW, Cheon JE, et al. Oribicularis oris muscle defects in philtral deformities in the repaired cleft lip. J Korean Soc Plast Reconstr Surg 2010;37:427-32. 